\title{
APRENDER HACIENDO CON OTROS. UN ESTUDIO DEL APRENDIZAJE INFANTIL EN EL MARCO DE LOS SISTEMAS DE ACTIVIDAD DE COMUNIDADES RURALES
}

\author{
Celia R. Rosemberg ${ }^{1}$, Bibiana Amado² \\ Consejo Nacional de Investigaciones Científicas y Técnicas (CIIPME-CONICET), Argentina \\ Universidad Nacional de Córdoba, Argentina
}

(Recibido el 01/10/2007, aceptado el 29/10/2007)

\begin{abstract}
RESUMEN
El presente trabajo estudia, en niños de comunidades rurales empobrecidas, el proceso de adquisición de conocimientos y habilidades involucrados en los sistemas de actividad, en tanto estos sistemas configuran los contextos interactivos donde se sientan las bases para el desarrollo lingüístico y cognitivo infantil. El corpus de datos está conformado por situaciones de interacción entre niños y adultos registradas durante la realización de actividades productivas familiares en las comunidades kollas rurales de Jujuy y de Salta y comunidades rurales de Córdoba, Argentina. Todas las situaciones fueron audio o video registradas. Se realizó entrevistas a niños y a adultos con el objeto de obtener información complementaria. Los resultados obtenidos mediante un procedimiento de análisis cualitativo, el Método Comparativo Constante (Glasser y Strauss, 1967; Strauss y Corbin, 1991), mostraron los diferentes modos en los que los niños se aproximan a los sistemas de actividades: modos en los que predominan las exploraciones lúdicas de los recursos u objetos propios de un sistema; modos caracterizados por la práctica de habilidades relacionadas con la actividad y modos que involucran la consecución de acciones específicas en el contexto de las actividades del sistema.
\end{abstract}

Palabras clave: Aprendizaje infantil, Actividad, Interacción social.

\begin{abstract}
This study analyses the processs by which children from poor rural communities acquire knowledge and abilities involved in their communities' activity systems. These systems are the interactive contexts in which children's language and cognitive development take place. The data consists of interaction situations between children and adults while families were carrying out productive activities in kolla communities from Jujuy and Salta and rural communities from Cordoba, Argentina. Complementary data were obtained by interviews of children and adults. The data were analysed with a qualitative procedure, the Comparative Constant Method (Glasser y Strauss, 1967; Strauss y Corbin, 1991). Results show the different ways

1 Investigadora del Consejo Nacional de Investigaciones Científicas y Técnicas (CIIPME-CONICET) Argentina. Profesora de la Facultad de Filosofía y Letras de la Universidad de Buenos Aires, Argentina. E-mail: crrosem@hotmail.com

2 Investigadora del Centro de Investigaciones Lingüísticas (CIL) de la Facultad de Lenguas de la Universidad Nacional de Córdoba, Argentina. E-mail: bibyamado@gmail.com
\end{abstract}


in which children became involved in the activity systems: exploring systems' resources and objects through play; practicing abilities related to the activity and participating in specific actions in the context of the activity.

Keywords: Children learning, Activity, Social interaction.

\section{INTRODUCCIÓN}

En todas las culturas los niños y las niñas aprenden haciendo, mirando, escuchando y jugando en situaciones compartidas con adultos y otros niños de su entorno social. Existen diferencias entre los niños de diferentes culturas con respecto a qué aprenden y en las estrategias por medio de las cuales lo hacen. Estas diferencias están vinculadas con los instrumentos, las condiciones de vida y los objetivos que se plantea cada grupo social.

En efecto, desde una perspectiva socio-histórico-cultural del desarrollo del hombre, se considera que el aprendizaje tiene lugar en el marco de la participación de los niños y las niñas en actividades que, en tanto propias de la especie humana, se caracterizan por estar mediadas por instrumentos psicológicos (Vygotsky, 1964; 1988; Bruner, 1986; Rogoff, 1994; Wertsch, 1991, 1998; Cole, 1999; Álvarez y del Río, 1999, 2001; del Río y Alvarez, 2002; Nelson, 1996). Estos instrumentos, entre los cuales se destacan los signos, implican una re-presentación de los estímulos que posibilitan al hombre operar mentalmente, esto es, sin que el objeto se halle material y externamente presente. Los instrumentos son el resultado de un proceso de construcción histórica, mediante el cual un grupo social hace frente a las restricciones del ambiente de un modo que asegura la supervivencia de los individuos y del grupo social como tal.

A través de su participación en los sistemas de actividad, los niños interiorizan los instrumentos de mediación propios de su cultura. Como señala Wertsch $(1988 ; 1991 ; 1998)$, Vygotsky concebía a la actividad externa en términos de procesos sociales mediatizados semióticamente. En el marco de este planteamiento, la internalización del discurso es la clave para comprender la transformación de los procesos sociales en fenómenos psicológicos, la clave para explicar la transición del funcionamiento interpsicológico al funcionamiento intrapsicológico. Se trata de un proceso de creación del plano interno de la conciencia, resultado de la participación del niño en actividades en cuya textura está incrustado el lenguaje.

Como señalan Álvarez y del Río (2001), cada cultura proporciona un modelo de vida, esto es, sistemas de actividades para sobrevivir y vivir, para construir la mente y la conciencia de un determinado modo. De ahí que el estudio del desarrollo infantil requiera atender a la cultura en el que éste se produce, a las dimensiones que definen los sistemas de actividades -objetivos, personas, objetos, creencias, valores, conocimientos, modos de enseñar y aprender, patrones de interacción, usos del lenguaje- y al interjuego de estas dimensiones con la adquisición del lenguaje y con el desarrollo de las estructuras, procesos y representaciones cognitivas.

El desarrollo cognitivo y el desarrollo lingüístico guardan entre sí una relación de incidencia recíproca. A partir de la convergencia actual de las teorías socioculturales de la enseñanza y el aprendizaje con los desarrollos de la psicología cognitiva (Nelson 
1996; Van Dijk, 1997), el discurso adquiere un rol relevante en el desarrollo cognitivo. En la matriz de aprendizaje, que configuran los sistemas de actividad, tiene lugar una construcción colaborativa de conocimientos en la que el rol del lenguaje, en tanto sistema representacional, no se limita sólo a cumplir funciones comunicativas externas sino también funciones cognitivas (Wertsch, 1998; Nelson, 1996).

Los trabajos que han estudiado los sistemas de actividad, en tanto contextos creados por las personas para relacionarse con la naturaleza y con la sociedad, han identificado diferentes categorías de elementos -tipo de entorno, recurso, técnica y finalidad- que configuran estos sistemas (Gallegos, 2001; Gasché, 2001) y han descripto los valores que cada una de estas categorías adquiere en actividades particulares (Amado y Borzone; 2007).

Los sistemas de actividad en los que participan los niños constituyen contextos que son controlados por los adultos a partir de la actualización de sus propios "guiones" (Shank y Abelson, 1977). Los "guiones" son representaciones cognitivas de la configuración de elementos en una actividad determinada. En la medida en que dan cuenta del modo en el que debe llevarse a cabo una actividad, imponen restricciones sobre las acciones de cada uno de los participantes (Nelson, 1996; Cole, 1999). Los guiones tienen una entidad doble: son tanto representaciones individuales como representaciones sociales compartidas. En efecto, son recursos para la interpretación idiosincrática que cada persona tendrá de la actividad conjunta y para la coordinación necesaria para reproducir esa actividad con otros. La comprensión y conformación de los guiones es central en el proceso de adquisición de la cultura (Cole, 1999), proceso clave para la psicología cultural (Nelson, 1996; Cole, 1999; del Río y Álvarez, 2002), que postula una visión de la cultura como proceso cognitivo que tiene lugar tanto dentro como fuera de la mente de las personas.

La mediación instrumental e interpersonal del adulto que coopera con el niño en una actividad conjunta permite que éste ejerza funciones superiores sin dominarlas: se trata de funciones socializadas y "prestadas" en la "zona de desarrollo potencial" (Vygotsky, 1964). El niño o la niña disfrutan de una conciencia im-propia, de una memoria, de una atención, de unas categorías, de una inteligencia que se hallan "distribuidas" en las actividades que comparte con el adulto. Ellas suplementan y conforman paulatinamente su visión del mundo y constituyen poco a poco su mente, que será, durante bastante tiempo, una mente que funciona con apoyos instrumentales y sociales externos. Sólo a medida que esa mente externa y social vaya siendo dominada con maestría y se vayan construyendo correlatos mentales de los operadores externos, las funciones superiores se irán interiorizando e irán conformando la mente del niño.

Del Río y Álvarez (2002) sostienen que, como el concepto de zona de desarrollo próximo conduce a considerar de un modo más laxo las fronteras entre el organismo y el medio, es necesario plantear la continuidad entre una ecología cultural y una ecología de la mente. En esta perspectiva, la noción de zona de desarrollo próximo no sólo tiene implicancias en el desarrollo interno sino también en el desarrollo externo: las funciones internalizadas por el individuo van a promover nuevas funciones en su cultura, la recreación y el cambio cultural.

De este planteamiento se desprende que para poder estudiar los procesos cognitivos en desarrollo sea necesario investigar los contextos de crianza a que dan lugar los sistemas 
de actividades que caracterizan a las distintas culturas (Bronfenbrener, 1979). A este respecto, cabe mencionar las diferencias entre los sistemas de actividades de las culturas tradicionales y las culturas industriales urbanas. Mientras que las primeras suponen papeles y valores estables que no pueden transgredirse, las culturas industriales conllevan una mayor inestabilidad y un cambio constante tanto en los papeles de los individuos como en los valores. Las prácticas educativas de las culturas tradicionales, basadas en una imbricación total del niño en sistemas de actividades socialmente compartidas por los miembros de una comunidad, han sido descriptas en diversas investigaciones (Rogoff, 1993; Cole, 1999; Rosemberg y Borzone, 1998; Borzone y Rosemberg, 2000).

Asimismo, se han comparado las consecuencias de las pautas sociales de comunidades tradicionales agrarias y modernas industriales sobre la vida de los niños (Álvarez y del Río, 2001). Rogoff, Paradise, Mejía Arauz, Correa Chávez y Angelillo (2003) han estudiado dos tradiciones básicas en los estilos de enseñanza y aprendizaje, que mantienen cierta correspondencia con los estilos propios de una y otra comunidad: el modelo de "participación intensa e intencional", según el cual los niños desarrollan aprendizajes mediante la realización de tareas familiares, y el modelo de "línea de montaje o fabril", que se caracteriza por un estilo de aprendizaje fragmentado y altamente desvinculado del contexto. En las comunidades rurales tradicionales la estructura de la participación de los niños y los adultos, los roles de los participantes, la motivación y el propósito de los aprendizajes, las fuentes de conocimiento y las formas de comunicación, todos estos aspectos permiten delimitar de modo más preciso los sistemas de actividad y la integración de los niños en tales sistemas.

En el marco de este planteo es posible pensar que el estudio de los modos de aprendizaje de los sistemas de actividad de comunidades rurales permite realizar contribuciones a un proceso de intercomprensión cultural (Gasché, 2001). El presente trabajo tiene, precisamente, por objeto estudiar, en niños de comunidades rurales empobrecidas, el proceso de adquisición de conocimientos y habilidades involucrados en los sistemas de actividad, que configuran los contextos interactivos donde se sientan las bases para el desarrollo lingüístico y cognitivo de los niños.

\section{MÉTODO}

\section{Recolección de información empírica}

El corpus de datos de este proyecto está conformado por situaciones de interacción entre niños y adultos registradas durante la realización de actividades productivas familiares en las comunidades kollas rurales de Jujuy y de Salta (74 situaciones) y en comunidades rurales de Córdoba (73), Argentina. Todas las situaciones fueron audio o video registradas.

Además, se realizaron 18 entrevistas a niños y 17 a adultos con el objeto de obtener información complementaria relativa a la ejecución de las actividades y sobre el modo de aprendizaje de tales prácticas familiares. Las entrevistas fueron audiograbadas. 


\section{Análisis de información empírica}

Se empleó un procedimiento cualitativo, el Método Comparativo Constante (Glasser y Strauss, 1967; Strauss y Corbin, 1991), para el análisis de los datos. Este procedimiento tiene por objeto generar, en el marco de un interjuego entre teoría y empiria, un sistema de categorías conceptuales que permitan comprender el fenómeno en estudio. Las categorías se desarrollan a través de un proceso inductivo en el que conjuntamente se codifica la información y se la analiza. El sistema de categorías elaborado da cuenta de los procesos de aprendizaje considerando los diferentes modos en que los niños se van aproximando a la actividad:

a) Exploraciones lúdicas de los recursos, objetos y acciones incluidas en los sistemas de actividad;

b) Ensayos de técnicas propias de la actividad;

c) La práctica avanzada que implica el dominio de las acciones del sistema de actividad.

En los resultados se muestra cómo estas categorías se despliegan en la interacción, ilustrando cada una de ellas con el análisis de casos ejemplares. La forma de presentación responde a las características metodológicas de los estudios comprensivos, que intentan acercar al lector la realidad concreta que conoció el investigador. El análisis conlleva la elaboración de los datos respetando su inserción original en un contexto complejo y la presentación de los rasgos más salientes en una descripción que reproduce para el lector su cualidad singular (Geertz, 1973; Gibaja, 1988). De este modo, por medio del análisis de ejemplos, de transcripciones de episodios y de citas textuales se intenta una aproximación al ideal de "descripción densa", que Geertz elevó a paradigma del modelo.

\section{RESULTADOS}

Los resultados del trabajo ponen de manifiesto cómo la estructura social de las comunidades rurales y la forma de organización de las actividades determinan que el aprendizaje infantil tenga lugar a través de la observación y de la participación de los niños en las situaciones de trabajo de los adultos. El poder de los padres y otros agentes de socialización reside en su capacidad de asignar a los niños a un escenario específico, por ejemplo, cuidando a un hermano pequeño, trabajando en el hogar con mujeres adultas, realizando tareas para la economía de subsistencia jugando con otros niños o asistiendo a la escuela. De este modo, los cuidadores guían tácitamente el desarrollo infantil.

El análisis de los registros de actividades de trabajo compartido entre niños y adultos muestra, por una parte, cómo los adultos y los niños mayores estructuran la participación de los niños más pequeños en las actividades. Pero, también, pone de manifiesto que esta regulación de la actividad, realizada por el adulto, se produce en forma articulada con acciones e iniciativas que realizan los niños y que revelan su interés y sus esfuerzos por integrarse a la situación.

La ejecución de la actividad en colaboración se caracteriza por la guía que proporciona el adulto, o un niño más experto, hacia la meta general u objetivo de la actividad y la focalización de la atención y de las acciones de los niños en los pasos que se deben dar para 
alcanzar las sub-metas de la tarea. De este modo, los mayores asumen la responsabilidad de organizar y deslindar las acciones que conducen al logro del objetivo. La incorporación de conocimientos que tiene lugar en estas situaciones y su organización en guiones -esto es, en representaciones mentales conformadas por una sucesión de acciones relacionadas- se hace evidente no sólo en la organización de la tarea por parte de los mayores y en las expresiones verbales que ellos emplean para regular, planificar y reconstruir imaginativamente la acción sino también en los juegos de los niños más pequeños y en sus progresivas acciones en el marco de la actividad pautada por los adultos,

Atendiendo a la participación de los niños, foco del presente análisis, las situaciones de interacción pueden caracterizarse teniendo en cuenta el modo en el que el niño se aproxima predominantemente a la actividad. Tanto en las comunidades kollas del noroeste de Argentina, como en las comunidades rurales de Córdoba, los niños manifiestan modos de aproximación similares: modos en los que predominan las exploraciones lúdicas de los recursos u objetos propios de un sistema -fibras de palma, lana, cabras-, modos caracterizados por la práctica de habilidades relacionadas con la actividad y modos que involucran la consecución de acciones específicas en el contexto de las actividades del sistema, mostrando un dominio significativo de las acciones del mismo.

Los niños pequeños suelen explorar los recursos que los adultos o los hermanos mayores emplean en las actividades familiares. Progresivamente, esa exploración, sin perder su componente lúdico, va integrando ensayos iniciales, esto es, acciones que tienen como finalidad el desarrollo de distintas destrezas necesarias para realizar la actividad de manera eficiente. Por su parte, en los aprendizajes avanzados, si bien el juego puede estar presente, las formas de aproximación están estrechamente vinculadas con las metas de la actividad del adulto.

Aún cuando no existe una correspondencia entre la edad de los niños y los modos en los que ellos se aproximan a la actividad, los registros muestran que, generalmente, los niños más pequeños observan a los adultos y, por medio del juego, exploran la actividad. Sin embargo, hay casos en los que a edad temprana comienzan a practicar algunas acciones dentro del guión de la tarea del adulto.

\section{El juego como modo inicial de aproximación a la actividad de trabajo}

En las primeras aproximaciones a una tarea, que son de carácter eminentemente lúdico, el niño explora las características de los recursos relacionados con la actividad. Por su parte, el adulto define las acciones permitidas o prohibidas de acuerdo con el riesgo que ellas pueden significar para los niños o con las interferencias que los juegos de los niños imponen a la realización de su actividad. Para ello, el adulto se vale de órdenes y de expresiones de control con el fin de regular verbalmente la acción de los niños.

Así, por ejemplo, durante la realización de actividades de cestería con hojas de palma, en una comunidad rural de Córdoba, los niños exploran los objetos que son parte de la actividad: las fibras de palma que emplean los tejedores de cestos. Con frecuencia se observa que los niños desordenan los haces de palma al jugar, tal como se advierte en los siguientes ejemplos: 
(1) Sandro (1: 6 años), mientras su madre teje, juega con los haces de palma.

Mamá: No me agarre la palma, hijo.

(2) Una tejedora sostiene en una entrevista:

Mi niño por ahí hace unos nudos o va y me hace enriedos en los tejidos míos, te digo, porque busca tejer. O por ái me saca toda la palma y me hace un desastre. $Y$ le digo: "Sos muy chiquito todavía, dejá de molestar con la palma". Es tremendo mi niño. ${ }^{3}$

En algunas de estas situaciones iniciales se observa que las acciones de parte de los niños son recontextualizadas por parte de la madre e incluidas dentro de la actividad como una parte del guión de la actividad que se está desarrollando. En estos casos, el lenguaje tiene un papel central para conectar significativamente las acciones del niño con la actividad del adulto. En el ejemplo que se presenta a continuación entre Sandro (1:6 años) y su madre, la exploración de las fibras de palma que lleva a cabo el niño es retomada y resignificada por las palabras de la madre en un nuevo contexto: el niño pequeño está ayudando a su madre a tejer.

(3) La madre ayuda con el tejido a una de sus hijas. Se acerca Sandro, que estaba jugando con unas hojas de palma

Sandro: Mamá, mamá.

Mamá: ¿Qué, hijo?

(Sandro le da palma).

Mamá: Gracias. Mi hijo me va a dar palma para tejer, ¿eh, Sandrito?

(Sandro sonríe).

La madre de Sandro interpreta la acción del niño proporcionándole por medio de su intervención una intencionalidad funcional en el marco del sistema de actividad -Gracias. Mi hijo me va a dar palma para tejer, ¿eh, Sandrito?-. Estas exploraciones iniciales, en las que son los adultos quienes crean el marco de significado para las acciones del niño, se continúan en situaciones en las que el contexto de significado es construido por los niños. Como se observa en el siguiente fragmento de intercambio, se trata de juegos regulados por los mismos niños en los que el lenguaje tiene también un papel relevante en el modo de estructurar la situación.

(4) Mario (3: 11 años) y Mirta (5; 4 años) de una comunidad kolla de Salta van a la chacra. Se sientan en la tierra mientras Francisco, su padre, está regando la tierra. Mario toma el pico y empieza a imitar a su padre.

Papá: Mario dejá eso. Te vas a lastimar.

Mario: No, papá.

(Mario sigue jugando con el pico)

3 Las transcripciones de los intercambios presentados a modo de ejemplo se han realizado manteniendo las formas fonológicas, léxicas, sintácticas y discursivas propias de las variedades del español que se habla en las comunidades en estudio. 
P: Hacé caso, chango.

M: No, papá, yo toy trabajando.

En estas aproximaciones lúdicas los niños manifiestan una comprensión inicial del vínculo entre los instrumentos - pico- y la consecución de la actividad -yo 'toy trabajando-. Sin embargo, es importante señalar que aquello que sostiene la acción pretendida en el marco de estas situaciones de juego no es el objeto o instrumento sino la representación del evento -guión- que el niño ya ha construido. Así por ejemplo, en el registro siguiente, tres niños kollas, Osvaldo (6: 4 años), Francisco (5: 2 años) y Gabriel (3: 11 años) juegan a sembrar sin recurrir a los instrumentos de los que se valen los adultos en la consecución de la actividad.

(5) Gabriel hacía de buey, araba la tierra en cuatro patas. Osvaldo tiraba la piola de adelante y Federico con una rama empujaba de atrás marcando la tierra y decía que estaban arando para sembrar las habas

Gabriel: Tá arando la tieya

Francisco: Nosotros sembrar con el agua

Gabriel: Tirá pues, el buey está apurao, corre,corre

Francisco: Es que el Osvaldito no tira bien pues

Gabriel: Ontonce no no are Osvaldito, el buecito quiere para sembrar yapidito las habas

(En ese momento Gabriel que era el buey sale corriendo y dice que quiere tomar agua)

Francisco: Eh! chango, se enojao el buey, corramo pa enlazar, corra, corra pa pillar!

Gabriel: El bueycito tiene hambre quiere tomar agüita y pastito con bramita.

(Federico es el hermano mayor, trae muchos yuyos secos para que el buey coma)

Gabriel: Bu, bú, bú, (dice Gabriel simulando ser el buey), ay! mi pancita esta llenao, no pede caminar

(Osvaldito va a traer agua en un bidón y comienza a tirar en la tierra)

Osvaldito: Changos tirima agüita, pa qui salga grandi la plantita

Francisco: No, primerito tenes que hacé la huellita pa tirá la agua, ontonce echá la agüita, no pues no sentojé, no sentoje el agüita

En el juego de los niños se advierte, por una parte, cuán completa es la representación mental, el guión que han internalizado de la actividad. Están presentes todos los elementos -buey, habas, agua pasto, huellita, tierra-y las acciones involucradas -sembrar, tirar del buey con una piola, tirar agua-. Asimismo, en las intervenciones finales de Osvaldito y Francisco- Osvaldito: Changos tirima agüita, pa qui salga grandi la plantita, Francisco: No, primerito tenes que hacé la huellita pa tirá la agua, ontonce echá la agüita, no pues 
no sentojé, no sentoje el agüita- se muestra el papel del juego, y también del lenguaje para crear una zona de desarrollo potencial en el marco de la cual, con la colaboración de los compañeros, se expanden y aclaran las representaciones mentales que subyacen a la situación de juego.

En efecto, en sus esfuerzos por contribuir al progreso de la trama de juego, los niños se valen de sus habilidades lingüísticas y comunicativas para establecer vínculos semánticos que implican extender, explicar y planificar los aportes de los otros participantes. Al hacerlo, los niños tienen la oportunidad de reconstruir colaborativamente la estructura narrativa de los eventos.

En la reconstrucción de la actividad los niños recuperan tanto su secuencia temporal -No, primerito tenés que hacé la huellita pa tirá la agua, ontonce echá la agüita-, como las relaciones causales incluidas en el guión -tirima agüita, pa qui salga grandi la plantita-. También atienden a lo que Bruner (1986) denomina el plano de la conciencia propio de las narraciones: los estados internos y las motivaciones de los personajes (el buey está apuao; se enojao el buey; el bueycito tiene hambre quiere tomar agüita y pastito).

\section{La práctica de habilidades y de destrezas relacionadas con las actividades}

Cuando los niños están familiarizados con la actividad, pero aún son pequeños, colaboran en general en forma espontánea y poco sistemática. Normalmente son ellos mismos quienes inician y dan por finalizada su participación, que consiste, básicamente, en una serie de ensayos iniciales, vinculados a ciertas técnicas de manipulación de los recursos y de las herramientas implicadas en la actividad.

(6) En la cocina están Gabriel (3: 11 años) de una comunidad kolla y su mamá. Gabriel entra, toma un cuchillo y pide una papa para pelar.

Gabriel: Yo cortar, mamá, papa.

Mamá: Cuidado con cortarte el dedo, changuito.

Observadora: Qué guapo como ayuda a la mamá.

Gabriel: Olla, cocer mamá papa.

Como se observa en el fragmento de intercambio, los niños usan el lenguaje acompañando la realización del evento, como una forma de regular su propia acción y señalizando lingüísticamente a los demás su ingreso y su desempeño en la actividad. A través del lenguaje, los niños dan también cuenta de su conocimiento global de las acciones que involucra el guión de la actividad.

Es frecuente que los niños realicen acciones que, aún cuando muchas veces no son las que realiza el adulto, configuran un paso previo para las acciones de los expertos. Estas acciones son organizadas por los mayores de acuerdo a pautas compartidas socialmente, que conducen a los niños a desarrollar las habilidades necesarias para las tareas integradas en cada sistema de actividad. Así, por ejemplo, en el caso de la actividad de cestería con hoja de palma, cuando los niños tienen mayor dominio de su motricidad, comienzan a realizar trenzas con las hojas de palma. En estas situaciones, utilizan palma verde, no 
madura que, aunque no es la que emplean los adultos para tejer con fines comerciales, es más flexible y facilita los primeros intentos de tejer que realizan los niños. El trenzado de fibras de palma permite a los niños explorar la flexibilidad de la palma y las posibilidades de formar un tejido inicial, que no suele hacer el adulto. Esta fase, que es esencialmente de práctica, tiene un objetivo específico: el desarrollo de destrezas motrices, tal como se ha registrado tanto en las observaciones como en las entrevistas.

(7) En una entrevista, una tejedora relata:

Las nenas empezaron tejiendo trenzas. Yo les enseñé con la palma bien verde. Verde verde, directamente. Para que no me echaran a perder la palma. Porque no les sale bien primero, entonces, "ustedes tejan con palma verde, les digo, hasta que se acostumbren y ya cuando salga mejor, les doy palma buena para que tejan”. Recién ái les doy la palma buena.

Es interesante señalar que la práctica para el desarrollo de habilidades complejas en tareas tales como el tejido de cestos con hoja de palma, en ocasiones, conlleva la consecución completa de la actividad hasta la elaboración de un producto. La elaboración es facilitada por la guía del adulto, quien crea un marco especial poniendo a tejer al niño en un lugar cercano a donde él teje, realizando la primera parte del tejido y proporcionándole al niño hoja de palma verde, que es más flexible. El producto elaborado -el cesto-, por las condiciones mismas que implica su producción asistida y facilitada por la palma verde tiene un carácter de "prueba", no se puede vender. Se trata de una actividad diseñada por los adultos para que los niños aprendan y, aún cuando está ligada al sistema de actividad productiva del adulto, se diferencia de ésta por su finalidad: no es la elaboración de un producto para su comercialización sino el aprendizaje del proceso de elaboración. En el siguiente fragmento una artesana explica el modo en que ha enseñado a tejer a sus hijas:

(8) La artesana está tejiendo un cesto. Sus hijas, de 6 y 7 años tejen cerca de ella cada tanto levantan la vista de su tejido y desvían la mirada hacia donde se halla la madre.

Artesana: Al principio yo les enseño con palma verde. Como esa es blanda no es para vender. Pero van aprendiendo. (Señala con un gesto a las nenas) Ellas tejen pruebas. Ese es el gusano, que le decimos (la madre señala el tejido de la niña). La primera parte se las hacemos para que les quede justo y no se le salgan los puntos. Y ya después ellas siguen.

\section{La consecución de acciones en el contexto de las actividades de los adultos. Hacia el logro de la autonomía en el desempeño}

Progresivamente, los niños comienzan a realizar acciones en el marco de las actividades propiamente adultas. Los adultos y los niños mayores guían las prácticas de los aprendices modelando las acciones y proporcionando señalamientos verbales. En las interacciones niño-adulto se regulan verbalmente las acciones y se solicita y proporciona información pero de un modo muy contextualizado, anclado en la actividad en curso, que estructura la comunicación. 
(9) Mabel, (6: 4 años) de una familia kolla de Jujuy está en el corral de las ovejas junto a su madre Doña Simona.

Madre: Mmm, poquito quiere tomar, este ha nacido esta mañana. ¿Cuál es el otro que ha parido la oveja, cuál es su mamá?

Mabel: Aque'stá.

Madre: Este pillá, este que ha parido ayer, este vamos a pillar.

Mab: Onde'ta pue ¿cuál es?

Madre: D'ese que se ha muerto, ese es su mamá, pillale!

Mabel: ¿Cuál es?

Madre: La blanca es

Mabel: ¿Esto, esto, mami? (Persigue a la oveja y la retiene hasta que llega su mamá)

Madre: Agarralo, agarralo!

(Doña Simona, la madre, agarra la oveja grande y se monta sobre ella, con una mano le sostiene las manos y con la otra sostiene al corderito recién nacido para que pueda entrar debajo de la oveja y pueda mamar algo. Doña Simona sostiene a ambas ovejitas todo el tiempo hasta que terminan de mamar, mientras Mabel le ayuda a sostener a la ovejita pequeña)

Madre: Andá hace mamar a la chitita... la choca (se refiere a la rubia)

Mabel: ¿Onde está? (camina buscando entre las ovejas)...eh ¿la choca?, chitita, chitita, chitita (llama a la ovejita)

Madre: Ese es su mama, po, hacé mamar de ella, ya sabís ¿no?

Mabe: ¿Esta?

El hecho de que los niños trabajen no parece impedir las posibilidades de juego ya que ambas actividades aparecen entrelazadas cuando los niños, en etapas posteriores de dominio de la actividad, siguen imprimiéndole a esta un carácter lúdico, tal como lo muestra el siguiente ejemplo:

(10) Ernestina (6: 6 años) alimenta a los chanchos.

Ernestina: Ya parece que se ha muerto. Venga mi chanchito, tomá agüita. ¡Ay! pero esta agua está sucia, yo te voy a dar más limpita. Tomá, tomá po, tomá aquí, mirá, más limpita. Yo te voy a dar agüita con bircabonato. ¡Ay! estos chanchitos está derramando todo. No, no, no le quiten, ustedes están guapos. Tomá, tomá, vos, el otro día se ha sanado así, después se ha muerto al otro día. Mirá, mirá como están sus panzas. Che, no me muerdan. ¿Quién está mordiéndome de la pollera?

(Juega y pelea con los chanchitos. Mete en una batea a los chanchitos junto con ella)

Ernestina: Toy yendo en un camión lindo, yo también me dentro. 
Este entrecruzamiento entre juego y trabajo se observa en la mayor parte se las situaciones en las que los niños deben colaborar alimentando y cuidando los animales que son un medio para la subsistencia familiar. Así por ejemplo, en la actividad de cría de cabras, los niños atrapan a los cabritos o juegan a "domar" a las cabras como si fueran caballos.

\section{(11) Entrevista a una niña (8 años)}

Entrevistadora: ¿Cómo hacés para sacar la leche?

Niña: Voy, agarro el tarro y veo si agarro alguna cabra. Y agarro pocas, tres o cuatro. Después le saco un poquito 'e leche y la echo al balde.

/.../

Niña: Una vuelta estábamos domando una cabra overa y mi hermana, la Noemí, subió adelante y yo subí atrás. Estábamos yo y ella. Y ella agarró una varillita y le pegó a la cabra, y hacía ¡meeeeee! hacía la cabra.

(risas)

Niña: Y la cabra corcovió y caímos al suelo.

Entrevistadora: ¿Se golpearon?

Niña: No.

Entrevistadora: ¿Así que ustedes suben a las cabras?

Niña: Sí. Las domamos. Ahora ya no.

Entrevistadora: Ahora ya no, ¿por qué?

Niña: Porque, cuando vienen las cabras, está un hombre, que le decimos Don Vera. $Y$ ahí no domamos más porque él le dice al tío.

Entrevistadora: Ah. ¿Le cuenta al tío? ¿El tío no quiere que ustedes domen las cabras?

Niña: No. (ríe) Quiere que saquemos leche nomás.

Cuando los niños alcanzan un dominio adecuado de las destrezas relacionadas con cada actividad, sus acciones comienzan a ser complementarias de las acciones que realizan los otros familiares que participan en el sistema de actividad. Como los requerimientos de cada sistema son variados en su grado de complejidad, los niños pueden aún estar desarrollando las habilidades necesarias para una tarea dentro del sistema y manifestar un desempeño adecuado en otras. Frecuentemente, los adultos conocen las potencialidades de los niños $\mathrm{y}$, por ende, se ocupan de asignar las funciones de cada integrante durante al realización de la actividad familiar.

Así por ejemplo, cuando los niños han adquirido las habilidades necesarias para realizar ciertas tareas del tejido en palma, los adultos les confieren la responsabilidad de ciertas acciones, como recolectar palma -que requiere conocer la flexibilidad necesaria de las hojas- o tejer parte de los canastos. En el ejemplo (12), una artesana hace referencia al modo en que participa su hija mayor, mientras que, en el segundo ejemplo (13), un tejedor expone de qué modo su hijo mayor lo asiste en su tarea de tejer. 
(12) M: Las chicas también rayan palma. Magalí busca palma, también. Ella ya la conoce a la palma que usamos para tejer.

(13) P: Mi hijo, el Negro, él me ayuda, me hace el empiezo de los canastos. Cuando termina la base, yo sigo el tejido.

Estas actividades realizadas por los niños son valoradas y requeridas por los padres, en tanto contribuyen a la subsistencia familiar. Desde pequeños, ellos también relevan a los adultos en muchas acciones de cuidado y alimentación de los animales.

(14) Entrevista a un capricultor

Los chicos son los que más saben en el corral, quizás más que nosotros. Porque a ellos no se les despinta un cabrito de una cabra. Este cabrito es de esta, o de otro. Por ahí ni los marcamos. Porque ellos los conocen bien. Y participan mucho, ayudan mucho. Colaboran digamos en la tarde cuando hay que entregar, ellos van a alcanzar: "Estos cabritos son de tal cabra de "la Overa", "la picasa", "la castaña", o "la chilluda", "la astuda" o "el asta quebrada".

(15) Entrevista a un capricultor

Los chicos colaboran mucho. En la mañana cuando van a entregar la majada ellos agarran y ya saben que hay que encerrarlos en el chiquero. Saben qué cabrito se tiene que quedar y el que tiene que irse al campo. Y en la tarde, bueno, ya saben que hay que encerrarlas, si hay que hacer separar cabra en un corral o en otro, ellos ya saben cuál va a un lado, cuál va a otro. Y cuando hay que sacar leche, ellos por ahí empiezan a practicar hasta que empiezan a sacar. $Y$, como ser, los más grandes saben sacar leche todos.

Cuando los niños ya se desempeñan en forma independiente en la actividad, se observó que, en algunas situaciones, actúan sin mediación del lenguaje, con lo cual se puede pensar que están siguiendo los patrones que estructuran mentalmente las acciones en los guiones. De tal modo, éstos funcionarían prospectivamente como "planes" para la acción. Pero con frecuencia emplean el lenguaje de modo regulatorio, enunciando los pasos y las acciones involucrados en la actividad, de un modo que pone de manifiesto la internalización de representaciones mentales elaboradas sobre el evento.

(16) Ernestina (6: 6 años) prepara la comida y enuncia las acciones que tiene que realizar.

Ernestina: Ya está por hervir la olla pal perro, que hierva un poquito, después yo voy a echar arroz a la sopa, la grasa a todo, la sal, todo vua'echar

(17) Ernestina (6: 6 años) va con su perro Chiladito al gallinero y habla dirigiéndose al animal.

Ernestina: Anoche he cerrau, cuando ha dicho... me ha ido a decir el Antucho “'has dejau cerrado las gallinas?”... A ver yo vua ir a ver, voy, yo voy llevando la piola y vua ver si está abierto ¿quién habrá soltau?, abierto del todo está

El candau ché, yo estoy por poner el candau a este, voy a poner el candau. 
En el discurso de Ernestina que acompaña la acción de cerrar el gallinero se deja ver la regulación que su padrino Antolín ha realizado previamente de la actividad - me ha ido a decir el Antucho ¿has dejau cerrado las gallinas?- y cómo la niña lo ha internalizado - A ver yo vua ir a ver, voy, yo voy llevando la piola y vua ver si está abierto-. De este modo, en los aprendizajes infantiles que tienen lugar en el marco de los sistemas en los que participa el grupo familiar se pone claramente de manifiesto el carácter discursivo, social y distribuido del conocimiento necesario para la consecución de la actividad.

\section{DISCUSIÓN}

Los resultados de este trabajo proporcionan nuevas evidencias empíricas relativas a los estilos de aprendizaje que caracterizan a las culturas aborígenes o a los niños que crecen en las zonas rurales, tal como fueron descriptos por Rogoff (1993) en su investigación en las comunidades mayas y por los hallazgos de otros investigadores (Scribner y Cole, 1973; Tharp y Gallimore, 1991; Tharp y Yamahuchi,1994).

En todos estos trabajos se señala que en las comunidades no se suele separar a los niños de las actividades de los adultos. Por lo que ellos aprenden, observando y haciendo, en la comunicación que se establece a través de las palabras y las acciones en la realización conjunta de tareas cotidianas. Aprenden con la guía de un adulto con el que están, a menudo, ligados por una relación familiar o de afecto.

La participación del niño en estas situaciones se ve facilitada porque el objetivo, al que se orientan todas las acciones individuales, puede ser inferido sin dificultad del contexto de la situación; porque los adultos muestran o modelan con sus acciones el desempeño que se espera del niño y porque, en ocasiones, dividen la tarea en sus componentes principales, asignan al niño una parte y le señalan los pasos necesarios para hacerla. El aprendizaje está contextualizado, tanto porque se aprende haciendo como porque lo que se hace tiene una función evidente

El análisis realizado en el presente trabajo ha permitido identificar distintos modos de aproximación de los niños a las actividades productivas de los adultos. En el marco de estos procesos de aprendizaje contextualizado, el juego constituye el modo inicial de aproximación a la actividad, o bien en la forma de exploraciones lúdicas de los recursos o acciones del sistema -exploraciones que son resignificadas por los adultos atendiendo los componentes del sistema-, o bien en la forma de juegos dramáticos a través de los cuales los niños recrean las acciones del sistema de actividad.

Un segundo modo en el que los niños se aproximan a las actividades adultas se caracteriza por la práctica de habilidades que implican la manipulación de los recursos y/o de las herramientas implicadas en la actividad. Estas acciones infantiles tienen lugar coordinadamente con las acciones de apoyo y guía del adulto. En algunos casos, las acciones de ambos participantes conforman una actividad de enseñanza y aprendizaje, que aún cuando se deslinda de la actividad productiva, comparte el tiempo y el espacio y está integrada y es subsidiaria de la actividad propiamente adulta.

El tercer modo identificado de aproximación infantil conlleva un dominio significativo de las acciones del sistema en tanto involucra la consecución de acciones específicas en el 
contexto de las actividades de los adultos, ya sea llevando a cabo conjuntamente con ellos la tarea o realizando parcial o totalmente la actividad en forma independiente del adulto.

Es importante enfatizar el hecho de que cada uno de estos modos de aproximación infantil a las actividades no constituye, de ninguna manera, una estrategia general de aprendizaje a la que el niño recurre en un momento determinado de su desarrollo para encarar el conjunto de las actividades con las que se enfrenta. Por el contrario, dado que los sistemas de actividad difieren, entre otras cosas, en cuanto a la complejidad de las habilidades que requieren del niño y en cuánto al interés y a la necesidad que el adulto tiene de que el niño participe, son también diferentes los modos en los que los niños intentan acercarse a la actividad. Es la actividad y la matriz de interacción, que en ella se genera, aquello que pauta el desempeño cognitivo infantil, tal como se postula desde los diversos enfoques que comparten una concepción sociocultural del desarrollo humano.

En un sentido similar, aún cuando entre estos modos de aproximación infantil a las actividades se registra una cierta transición -desde la exploración de recursos y prácticas en marcos lúdicos hasta el desarrollo de habilidades más complejas y diversas, necesarias para alcanzar el dominio de la actividad- esta transición no implica una progresión a la manera de etapas en las que la aparición de un nuevo modo de aproximación conlleva la desaparición de los modos anteriores. Así por ejemplo, el juego, como modo inicial de aproximación a las situaciones de trabajo perdura en muchas situaciones de práctica avanzada, o bien como una atmósfera lúdica que impregna la actividad (Sarlé, 2006), o bien en la forma de recreaciones dramáticas o simbólicas de la situación, en las que la intencionalidad propia de la actividad productiva se entrelaza con un nuevo significado dado por el juego del niño.

En sus juegos iniciales los niños re-representan solos o con la colaboración de otras personas la actividad productiva de los adultos. En la textura de estos juegos, la representación se configura tanto a través de la dramatización de acciones como lingüísticamente. Paulatinamente, los niños progresan en sus habilidades para incorporar el juego y el uso del lenguaje como modos de representación cognitiva (Nelson, 1996) que les permiten reconstruir mentalmente su experiencia: los niños juegan a que trabajan, cuentan a otros que trabajan y transforman la situación de trabajo en situaciones de juego

Sin duda, el hecho de que los niños puedan enunciar verbalmente los pasos requeridos en la realización de una actividad determinada, ya sea para explicársela a otra persona o como una forma de regular su propia acción, muestra el desarrollo de conocimientos no solo procedurales sino también declarativos que involucra el dominio de esa actividad.

Asimismo, el hecho de que los niños puedan transformar la situación de trabajo en una situación de juego dramático pone de manifiesto que el guión (Nelson, 1996; Cole, 1999) que los niños han construido de la actividad no constituye una representación fija y rígida, como la que caracteriza a las primeras formas de conocimiento, sino una representación mental flexible que permite la manipulación mental de las acciones y de los instrumentos, en función de los objetivos de la actividad productiva y del objetivo del guión de la actividad que se quiere representar jugando (Nelson, 1996).

El estudio de estos diversos modos de aproximación infantil a las actividades adultas proporciona evidencias del carácter, también diverso, de los conocimientos que los niños 
adquieren fuera del ámbito escolar. Cada sistema de actividad integra una amplia gama de conocimientos sobre sus recursos -ya sean animales de cría, plantas que se usan con distintos fines, etc.- y sobre las técnicas y los procedimientos empleados, como formas de medición, cálculos mentales, el desarrollo de destrezas motrices y da lugar a un modo particular de acceso por parte del niño. Estos "fondos de conocimientos", en términos de Gonzalez, Amanti y Moll (2004), son significativos para el niño en dos sentidos: en tanto suponen una identificación con el medio social, por ser prácticas culturales construidas a lo largo de un proceso histórico, y en cuanto integran un sistema cuyas metas el niño conoce previamente. Sin embargo, en la escuela no siempre se consideran como fuentes de nuevos aprendizajes esos conocimientos, ni los modos de acceso a ellos, que los niños han adquirido y desarrollado en el medio familiar.

En efecto, se han identificado importantes diferencias entre el hogar y el aula en aspectos relativos a los procesos de construcción de conocimientos en cada uno de estos entornos. Se ha señalado que los "fondos de conocimiento" que los niños aprenden en su entorno familiar y comunitario no son habitualmente considerados en el entorno escolar (Moll, 1992; Trueba, 1999; Borzone y Rosemberg, 2000; Gonzalez, Amanti y Moll, 2005). Se ha mostrado, además, que la aproximación holística a situaciones reales, que caracteriza a los modos de acceso al conocimiento en el medio familiar, no es valorada en la escuela, que privilegia, en cambio, la comparación y el análisis conceptual (Rogoff, 1993; Cole, 1999).

Estos desajustes en la compleja interrelación entre el entorno familiar del niño y el entorno escolar explican, al menos en parte, los altos índices de fracaso escolar que se registran en niños de las comunidades rurales (Cook Gumperz, 1988; Michaels, 1988; Simons y Murphy, 1988; Wells 1985, Álvarez y del Río, 1991; Tharp y Gallimore, 1991; Tharp y Yamahuchi, 1994; Rosemberg y Borzone, 1998; Borzone y Rosemberg, 2000).

\section{CONCLUSIONES}

En el marco de esta problemática cobran relevancia los resultados obtenidos en el presente trabajo, en tanto se tornan evidentes sus implicancias pedagógicas. La escuela puede facilitar el "encuentro" de los niños con los conocimientos y habilidades que constituyen su objeto de enseñanza creando en el aula situaciones de enseñanza contextualizadas, situaciones en las que el juego sea una forma de acceso a contenidos académicos; en las que las actividades de práctica de habilidades complejas no se presenten de forma fragmentada sino que remitan a la significación que proporciona el conocimiento del objetivo de la actividad; situaciones en las que el dominio mayor de una actividad se acompañe de mayores y nuevas responsabilidades por parte de los niños; situaciones en las que, al estar ancladas en los conocimientos y problemas del entorno del niño, los géneros discursivos elaborados se enseñen y se aprendan como herramientas que posibiliten la reconstrucción lingüística de la propia realidad. Solo en este marco de enseñanza y aprendizaje los conocimientos, así como los modos de aproximación a los conocimientos que los niños han desarrollado en su entorno familiar y comunitario, se tornarán productivos para lograr nuevos aprendizajes. 


\section{REFERENCIAS BIBLIOGRÁFICAS}

1. Álvarez, A. and del Río, P. (1999). Cultural mind and cultural identity: Projects for life in body and spirit. Activity theory and social practice: Cultural-historical approaches. S. Chaiklin, M. Hedegard and U. J. Jensen. Aarhus, Aarhus University Press: 302-324.

2. Álvarez, A. y Del Río, P. (2001). "Culturas, desarrollo humano y escuelas. Hacia el diseño cultural de la educación". En Cultura y educación vol. 13. Madrid: EDISA.

3. Amado, B. y Borzone, A.M. (2007). Los sistemas de actividad en la construcción de puentes hacia los conocimientos formales. Trabajo inédito.

4. Borzone de Manrique, A.M. y Rosemberg, C. (2000). Aprender a leer y a escribir entre dos culturas. Buenos Aires: Aique.

5. Bronfenbrenner, U. (1987). La ecología del desarrollo humano. Buenos Aires: Paidós.

6. Bruner, J. (1986). El habla del niño. Barcelona: Paidós.

7. Cole, M (1999). Psicología cultural. Madrid: Morata.

8. Cook-Gumperz, J. (1988). La construcción social de la alfabetización. Buenos Aires: Paidós.

9. Del Río, P. y Álvarez, A. (2002). "From activity to directivity. The question of involvement in education". En G. Claxton \& G. Wells (Eds.), Learning for life in the 21st Century: Sociocultural perspectives on the future of education (pp. 59-72). Oxford, UK: Blackwell.

10. Gallegos, C. (2001). "El currículo de primaria basado en actividades como puente entre la cultura local y global: una experiencia educativa en la Amazonía peruana". En Cultura y educación vol. 13. Madrid: EDISA.

11. Gasché, J. (2001). "El reto de una educación indígena amazónica: alcances provisionales". En Cultura y educación vol. 13. Madrid: EDISA.

12. Geertz, C. (1973). The interpretation of cultures: Sellected Essays. New York: Basic Books.

13. Gibaja, R (1988). "Acerca del debate metodológico en la investigación educacional". Revista interamericana de desarrollo educativo (OEA).

14. Glasser, B. y A. Strauss (1967). The discovery of grounded theory. Chicago: Aldine Publishing Company.

15. González, L. Moll y C. Amanti (2004). Funds of knowledge: Theorizing practices in households, communities, and classrooms. Mahwah, NJ: Lawrence Erlbaum Associates.

16. Michaels, S. (1988). "Presentaciones narrativas: una preparación oral para la alfabetización con alumnos de primer curso". En Cook-Gumperz, J. La Construcción social de la alfabetización (pp. 109-135). Barcelona: Paidós. 
17. Moll, L.C. (1992). "Bilingual classroom and community analysis: Some recent trends". Educational researcher, 21, 2, 20-24.

18. Nelson, K. (1996). Language in cognitive development. The emergence of the mediated mind. Cambridge: Cambridge University Press.

19. Rogoff, B. (1993). Aprendices del pensamiento. El desarrollo cognitivo en el contexto social. Barcelona: Paidós.

20. Rogoff, B., Paradise, R., Mejía Arauz, R., Correa Chávez, M., y Angelillo, C. (2003). "Firsthand learning through intent participation". Annual Review of Psychology, 54, 175-203.

21. Rosemberg, C. y Borzone de Manrique, A.M.(1998). "Interacción verbal y cognición: el desarrollo de los niños collas en el entorno familiar y escolar". Lenguas modernas, 25:95-113.

22. Sarlé, P. (2006). Enseñar el juego y jugar la enseñanza. Buenos aires: Paidós.

23. Schank, R.y R. Abelson (1977). Scripts, plans, goals and undestanding. Hillsdale, NJ: Erlbaum.

24. Scribner, S. y Cole, M. (1973). "Cognitive consequences of formal and informal education". Science, 182, 553-559.

25. Simons, H. y S. Murphy (1988). "Estrategias en el lenguaje hablado y adquisición de la aptitud de leer”. En Cook-Gumperz J. La construcción social de la alfabetización. Barcelona: Paidós.

26. Strauss, A. \& Corbin, J. (1991). Basics of cualitative research. Grounded theory. Procedures and technics. London: Sage Publications.

27. Tharp, R.G. y Gallimore, R. (1991). The instructional conversation: Teaching and learning in social activity http://www.ncbe.gwu.edu/miscpubs/ncrcdsll/rr2.htm

28. Tharp, R.G. y L.A. Yamauchi (1994). Effective instructional conversation in native american classroooms http://www.ncbe.gwu.edu/miscpubs/ncrcdsll/epr10htm

29. Trueba, e.T. (1999). Latinos unidos: From cultural diversity to the politics of solidarity. MD: Roman y Littlefield

30. Van Dijk, T. (1997). "Towards a theory of context and experience models in discorse processing". En Herre Van Oostendordp (Comp.) The construction of mental models during reading. Hillsdale, N.J.: Erlbawm.

31. Vygotsky, L.S. (1964). Pensamiento y lenguaje. Buenos Aires: Editorial Lautaro.

32 Vygotsky, L.S. (1988). El desarrollo de los procesos psicológicos superiores. Barcelona: Grijalbo.

33. Wells, G. (1985). "Preschool literacy-related activities and success in school". En D. Olson, N. Torrance \& A. Hildyard (Eds.) Literacy, language, learning. The nature and consequences of reading and writing. New York: Cambridge University Press. 
34. Wertsch, J.V.(1988). Vygotsky y la formación social de la mente. Barcelona: Paidós.

35. Wertsch, J.V. (1991). Voces de la mente. Un enfoque sociocultural para el estudio de la acción mediada. Madrid: Visor.

36 Wertsch, J.V. (1998). La mente en acción. Buenos Aires: Aique. 